\title{
PÓS-GRADUAÇÃO EM ENGENHARIA MINERAL NA UFOP (UM RETROSPECTO)
}

\author{
F. SÃO JOSÉ , J. OSTI, F. C. NOGUEIRA, C. A. PEREIRA \\ Universidade Federal de Ouro Preto, Programa de Pós-Graduação em Engenharia Mineral \\ fabio.sao.jose@hotmail.com**
}

Submetido 02/11/2017 - Aceito 20/11/2017

DOI: $10.15628 /$ holos.2017.6496

\section{RESUMO}

Busca-se no Programa de Pós-graduação em Engenharia Mineral (PPGEM) da Universidade Federal de Ouro Preto (UFOP) oferecer um nível de ensino que se destaque no âmbito da formação de especialistas em Engenharia de Minas, com foco em Tratamento de Minérios ou Lavra de Mina. Tal programa iniciou-se em março de 1998, apoiando-se na tradição e seriedade dedicadas à formação de Engenheiros de Minas pela Escola de Minas/UFOP. Assim, busca-se, atualmente, a integração entre graduação, pós-graduação, pesquisa e extensão cujos seus docentes atuam também em atividades didáticas da graduação. Hoje, existem desafios a serem vencidos como a competição por recursos governamentais disponíveis, a busca de apoio empresarial e renovação nas bases idealísticas do ensino de pós-graduação. Portanto, esse artigo foi desenvolvido e destinará a ser uma ferramenta composta de dados levantados in situ, mas que servirá para o crescimento e melhoria, ou seja, renovação da pós-graduação na engenharia mineral na UFOP e em todo país.

PALAVRAS-CHAVE: Pós-Graduação, Engenharia Mineral, Engenharia de Minas, UFOP.

\section{MINERAL ENGINEERING POST-GRADUATE AT UFOP (RETROSPECT)}

\begin{abstract}
The graduate program in Mineral Engineering search offer a level of teaching that allows the training of experts in mining engineering, with a focus on Mineral Processing and Mining. Such program was initiated in March 1998, leaning on tradition and seriously dedicated to the formation of mining engineers by School of Mines/UFOP. Thus, currently, the Department aims to integrate undergraduate, postgraduate, research and extension faculty. Today, there are
\end{abstract}

challenges to be overcome as the competition for government resources available, the search for business support and renewal in the idealistic graduate education bases. Therefore, this article was developed and will be a tool comprised of data collected in situ, but which will serve for growth and improvement, namely, renewal of graduate in mineral engineering in UFOP and throughout the country.

KEYWORDS: Graduate, Mineral Engineering, Mining Engineering, UFOP. 


\section{APRESENTAÇÃO}

A proposta do Programa de Programa de Pós-graduação em Engenharia Mineral é oferecer um nível de ensino que se destaque no âmbito da formação de especialistas em Engenharia de Minas, com foco nas áreas de Tratamento de Minérios ou Lavra de Mina. Cria-se, dessa forma, a pontualidade em temas tais como pesquisa operacional aplicada a indústria mineral, modelos matemáticos em mecânica das rochas, controle do impacto ambiental produzido pela mineração, geotécnica ambiental, modelagem, simulação e controle de processo, tratamento de efluentes e flotação entre outros. O programa iniciou-se em março de 1998, apoiando-se na tradição e seriedade da Escola de Minas à formação de Engenheiros de Minas por mais de 138 anos.

O curso em Engenharia de Minas foi criado no final do século XIX e mostrou evolução em relação ao ensino praticado na época como destacam Roque (1999) e Schwartzman (2001) em suas revisões. A criação do curso disponibilizou e continua a disponibilizar profissionais para atender demandas de empresas do setor minero-metalúrgico, órgãos públicos, corpo docente, pesquisa, extensão entre outras atividades. Roque (1999) chama atenção para o papel que possuíam os ex-alunos na política brasileira no inicio do século $\mathrm{XX}$, atendendo locais como norte e nordeste. Porém hoje existem alguns desafios a serem vencidos: competição por recursos governamentais disponíveis, busca de apoio empresarial e a renovação do modelo de ensino no nível de graduação e pós-graduação, pois ambos não contemplam a formação docente como objetivo de importância.

Na Universidade Federal de Ouro Preto (UFOP), busca-se a integração entre a graduação, pós-graduação, pesquisa e extensão. Os docentes da pós-graduação atuam também em atividades didáticas na graduação, destacando-se a administração de disciplinas e co-orientações de projetos e trabalhos de conclusão de curso. Assim como na estrutura física do departamento competente, a qual é compartilhada entre ambos os níveis de ensino. Os temas das iniciações científicas (projetos de pesquisas com fomento) e dissertações visam buscar a solução de problemas industriais (parcerias escola-empresa), sendo investigados com base em metodologia científica, emprego de conceitos fundamentais e ferramentas laboratoriais e de informática (modelagem).

O pós-graduando depara-se no curso com uma proposta de formação profissional qualificada nos fundamentos e aplicações da Engenharia Mineral com visão crítica em relação ao desenvolvimento sustentável e suas implicações, preparando-se para atuar no sistema produtivo, em centros de pesquisa e na docência. Esse profissional se faz cada vez mais necessário, uma vez que se exige mais qualidade na atividade mineradora no país, que sofre com elevações de custos, quedas bruscas de preços, maiores exigências por clientela e fiscalizações ambientais.

Nos últimos anos observou-se um demandado crescimento de cursos de engenharia de minas no Brasil, que saiu de sete para vinte e nove cursos, no entanto há necessidade de se repensar na formação do engenheiro tanto na graduação quanto na pós-graduação, pois em nenhum deles se contempla atender a formação docente para o sustento da nova demanda criada. 
O primeiro Plano Nacional de Pós-graduação (1975-1979) buscava formar docentes e pesquisadores e ainda apresenta hoje a mesma preocupação do VI Plano Nacional de Pósgraduação (2011-2020). Esse último vai mais além, pois visa também a integração maior com a empresa e sociedade e não se esquece da formação de professores para os ensinos médio e básico. Se existe esse plano nacional, como as Pró-Reitorias de graduação têm articulado, junto a seus coordenadores de cursos de pós-graduação, para atendê-lo? Nota-se muito pouco a preocupação de programa de graduação e pós-graduação com a formação de novos docentes.

Portanto, elaborou-se esse trabalho que visa divulgar dados do Programa de PósGraduação em Engenharia Mineral da UFOP e iniciar discussões quanto seus objetivos, destacando-se a formação de novos docentes.

\section{MATERIAIS E MÉTODOS}

A metodologia empregada neste trabalho consistiu no levantamento de dados junto ao colegiado do atual programa de Pós-Graduação em Engenharia Mineral da UFOP com o objetivo de divulgar característica e dados históricos do curso. Utilizou-se a Plataforma Sucupira/ Coordenação de Pessoal de Nível Superior (CAPES) para consulta de dados atualizados.

Em primeiro plano apresentou-se um pequeno histórico do curso, retirado de registros produzidos pelo próprio (CPPGEM) Colegiado da Pós-Graduação e do Departamento de Engenharia de Minas (DEMIN) da UFOP;

Um segundo plano expôs o corpo docente que atua como professores credenciados e colaboradores a lecionar na pós-graduação;

Coletaram-se dados quanto ao ano da apresentação e conclusão do mestrado dos alunos de Engenharia de Minas da UFOP no período de 2000 até 2016, assim como números de produção científica.

\section{RESULTADOS E DISCUSSÃO}

A área que contempla lavra e tratamento de minérios possui hoje nove cursos de pósgraduação em todo país. São oferecidos três cursos no nível de mestrado e seis para doutorado que incluem a engenharia mineral. Entre tais cursos, hoje faz parte o Programa de Pós-graduação em Engenharia Mineral da UFOP (PPGEM-UFOP).

\subsection{PPGEM-UFOP de sua origem aos dias atuais}

A Tabela 1 resume os principais acontecimentos no programa de pós-graduação em Engenharia Mineral, o qual passou por algumas modificações até atingir hoje a inclusão do doutoramento.

Tabela 1 - Histórico do Programa de Pós-graduação em Engenharia Mineral (CPPGEM, 2017).

\begin{tabular}{c|c}
\hline 1998 & Criação do curso de Pós-graduação em Engenharia Mineral; \\
\hline 2000 & Ano das primeiras defesas (Título: Mestre em Ciências da Engenharia Mineral); \\
\hline 2013 & Ano de maior número de publicações em periódicos; \\
\hline 2014 & Obtenção do conceito CAPES 4; \\
\hline 2015 & Inclusão do curso de Doutorado em Engenharia Mineral. \\
\hline
\end{tabular}


O conceito-CAPES do programa foi elevado, pela própria CAPES em meados do ano de 2014. A nota foi elevada de 3 para 4 o que abriu caminho para a inclusão do curso de doutorado junto ao mestrado nas áreas de tratamento de minérios e lavra de mina. No entanto, a divulgação de edital para formação da primeira turma de doutorandos só foi lançado no segundo semestre de 2015 devido a necessárias adaptações prévias.

Com a finalização do primeiro concurso para doutorado, foram admitidos 9 discentes, sendo 4 para a subárea do tratamento de minérios e demais alocados em lavra de mina. Em 2016, após o segundo concurso, são 12 doutorandos no total, distribuídos nas citadas subáreas. Já até outubro de 2017 o número de doutorando alcançou a marca de 17 matriculados

\subsection{Docentes do PPGEM-UFOP}

O corpo docente do curso é composto por 12 professores, todos permanentes. Destes membros permanentes 7 são bolsistas de produtividade do CNPq, sendo o maior número entre os cursos de pós-graduação da área. A Tabela 2 lista os nomes dos professores e destaca seu nível de pesquisador.

Tabela 2 - Lista do corpo docente do Programa de Pós-graduação em Engenharia Mineral (CPPGEM, 2017).

\begin{tabular}{c|c|c}
\hline Docente & Bolsa Pesquisador CNPq & Categoria \\
\hline Adilson Curi & B2 & Permanente \\
\hline Carlos Alberto Pereira & B2 & Permanente \\
\hline Christiane de Lyra Nogueira & B2 & Permanente \\
\hline Érica Linhares Reis & - & Permanente \\
\hline Hernani Mota de Lima & B2 & Permanente \\
\hline Ivo Eyer Cabral & - & Permanente \\
\hline José Aurélio Medeiros da Luz & B2 & Permanente \\
\hline José Margarida da Silva & - & Permanente \\
\hline Milene Sabino Lana & B2 & Permanente \\
\hline Otávia Marins da Silva Rodrigues & - & Permanente \\
\hline Rosa Malena Fernandes Lima & B2 & Permanente \\
\hline Waldyr Lopes de Oliveira Filho & - & Permanente \\
\hline
\end{tabular}

\subsection{Pós-graduação em Engenharia Mineral em números}

A Figura 1 mostra, por meio de gráfico, o número de alunos do PPGEM-UFOP que concluíram o mestrado entre 1998 a outubro de 2017. 


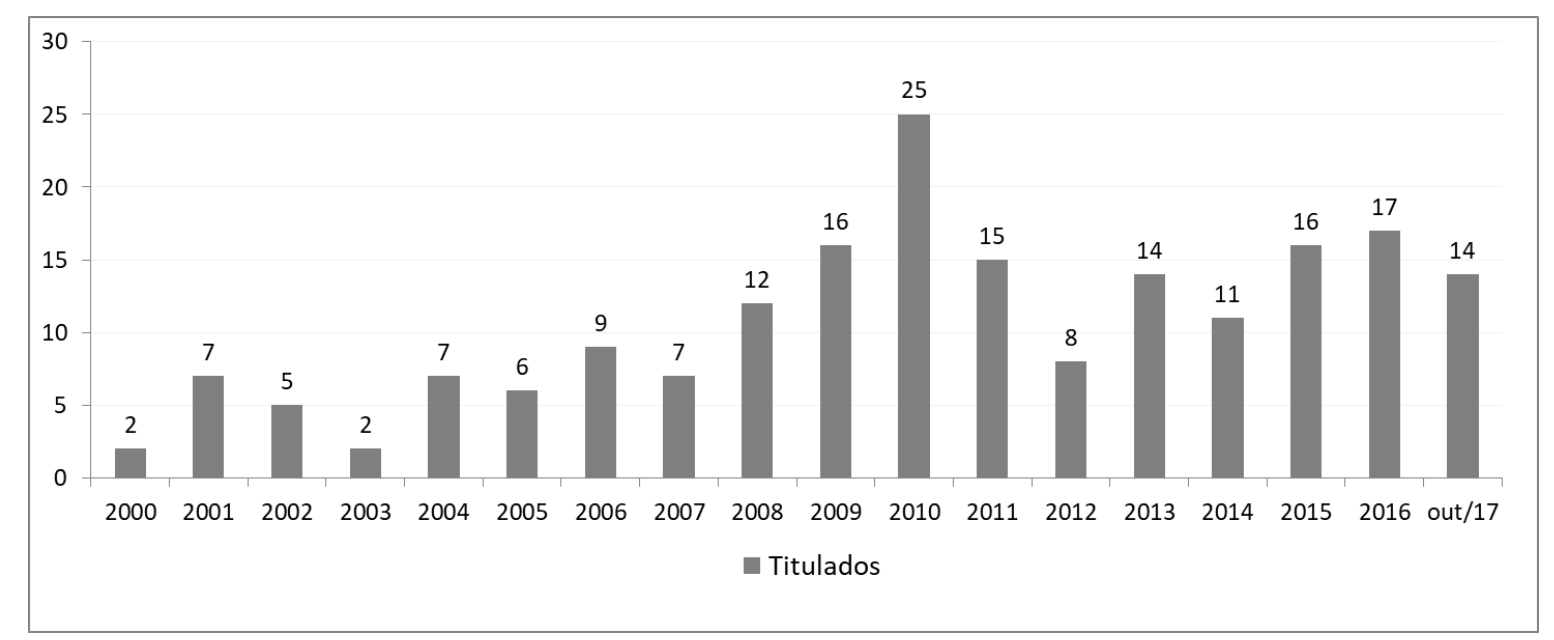

Figura 1: Quantidade de alunos que concluíram o mestrado na UFOP no período de 1998 a outubro de 2017 (Plataforma Sucupira/CAPES).

De acordo com a Figura 1, nota-se que de 2000 a 2007 não houve grandes variações no número de concluintes do curso, e período no qual não se ultrapassou a marca de dez formados. Esse período foi marcado por um mercado de trabalho estável, com pouca oferta de emprego e, portanto menor procura pela especialização na área correlata ao estudo.

A partir de 2008 notou-se um crescimento nas conclusões do curso, devido à percepção de um mercado mais exigente, com incentivo à busca por melhores qualificações e poucas ofertas de trabalho. Crescimento esse que durou até o ano de 2010, ano destacado com o maior número de conclusões, coincidente com o forte aquecimento do mercado de trabalho, com grande elevação dos preços de commodites (principalmente minério de ferro, como mostra a Figura 2). No entanto, a partir do ano seguinte (2011) registrou-se queda nas conclusões, voltando a patamares anteriores a 2008.

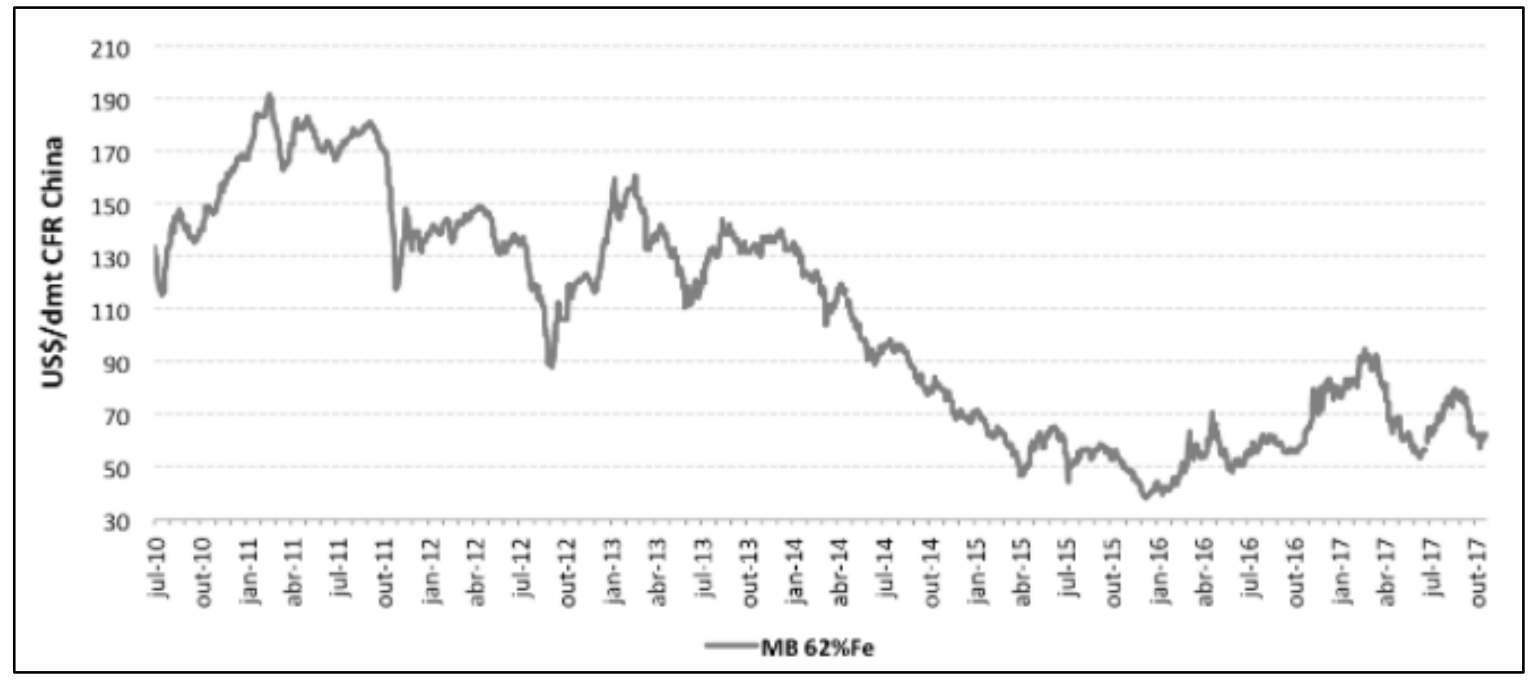

Figura 2: Índice de preço de minérios de ferro a vista (Adaptado de www.vale.com).

Novamente, a partir de 2013, houve crescimento do número de alunos titulados, conforme Figura 3, que apresenta também o número de alunos matriculados.

A procura pelo mestrado em Engenharia Mineral cresceu mais de $83 \%$ nos últimos quatro anos. Situação condizente com um período de poucas ofertas de trabalho e um mercado cada vez mais exigente que passa a considerar a pós-graduação como pré-requisito e não mais uma 
formação diferencial. O acumulo de matriculados dos anos de 2016 e 2017 certamente impactará em maior número de titulados nos próximos anos.

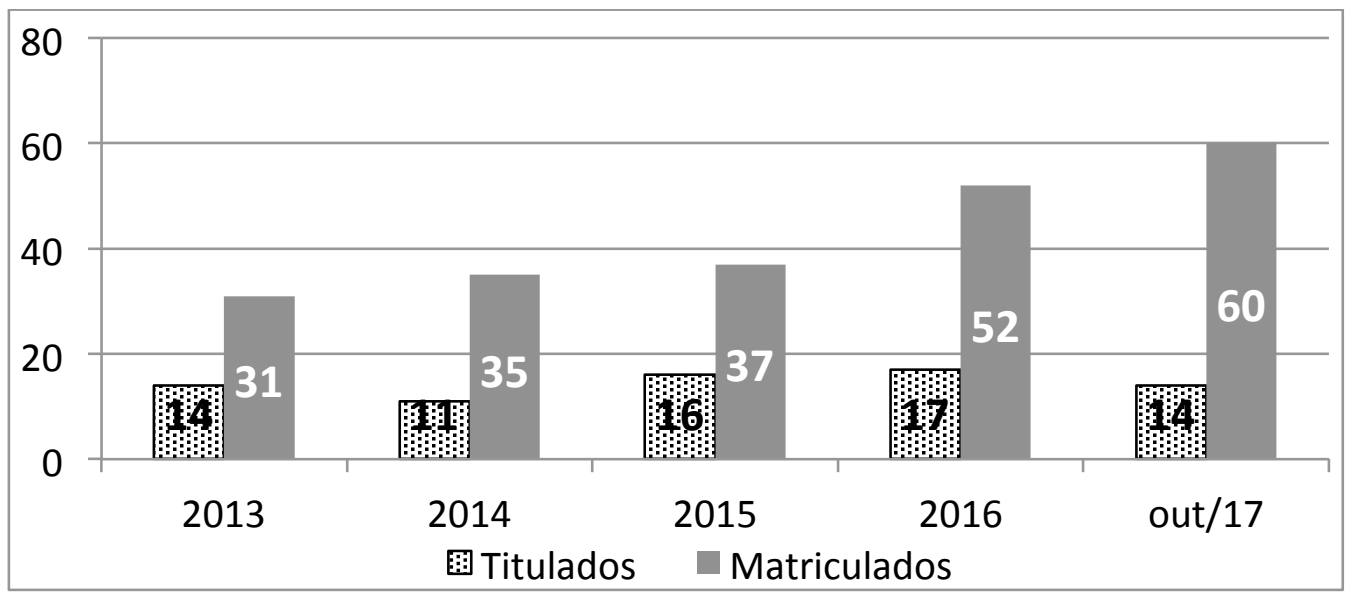

Figura 3: Comparativo de matriculados e titulados no período de 2013 a 2016 (Plataforma Sucupira).

A Figura 4 mostra o número de produção científica do curso de pós-graduação em Engenharia Mineral nos períodos antes 2010 e até o presente. Incluem na contagem de produção todos os trabalhos apresentados em eventos nacionais, assim como artigos científicos publicados em jornais e periódicos. A publicação é sem dúvida uma das atividades mais importantes da atividade acadêmica. Por meio da publicação de novos artigos, a comunidade acadêmica cria uma rede de novos assuntos, aproxima a academia da área industrial e serve como medida de produtividade para os pesquisadores.

Os critérios de classificação do periódico são definidos pela CAPES, sendo periódico A1 de maior nível, enquanto que periódicos B5 de menor nível nessa classificação. Não se encaixam nessa classificação os trabalhos divulgados em anais de eventos.

As publicações em periódicos B1 tem se destacado nos registros do PPGEM. A destinação quase que exclusiva de trabalhos à revista Escola de Minas, da mesma instituição de ensino na qual o PPGEM se integra, foi a justificativa para a ampla diferença de números de publicações por nível de periódico.

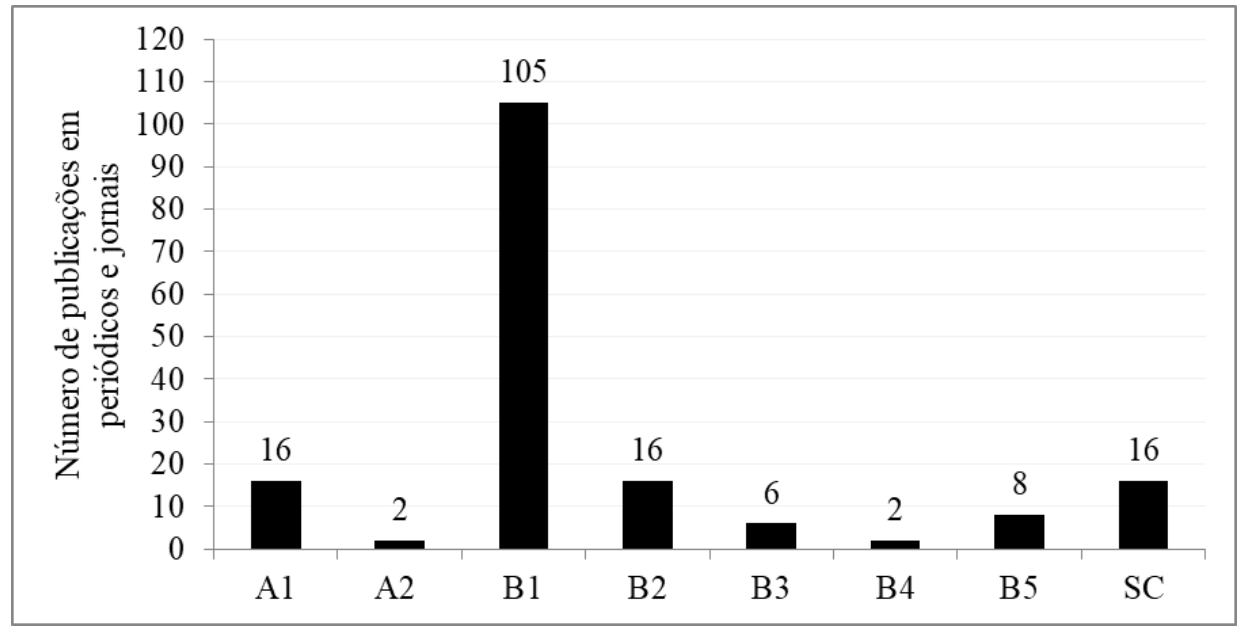

Figura 4: Produção bibliográfica do curso de pós-graduação em Engenharia Mineral (Plataforma Sucupira). 
A Figura 5 exibe a produção bibliográfica do curso de Engenharia Mineral separadamente entre professores e alunos. Como exigência à titulação, o aluno deve submeter seu trabalho a um veículo condizente com a área Engenharias II, não significando sua real publicação.

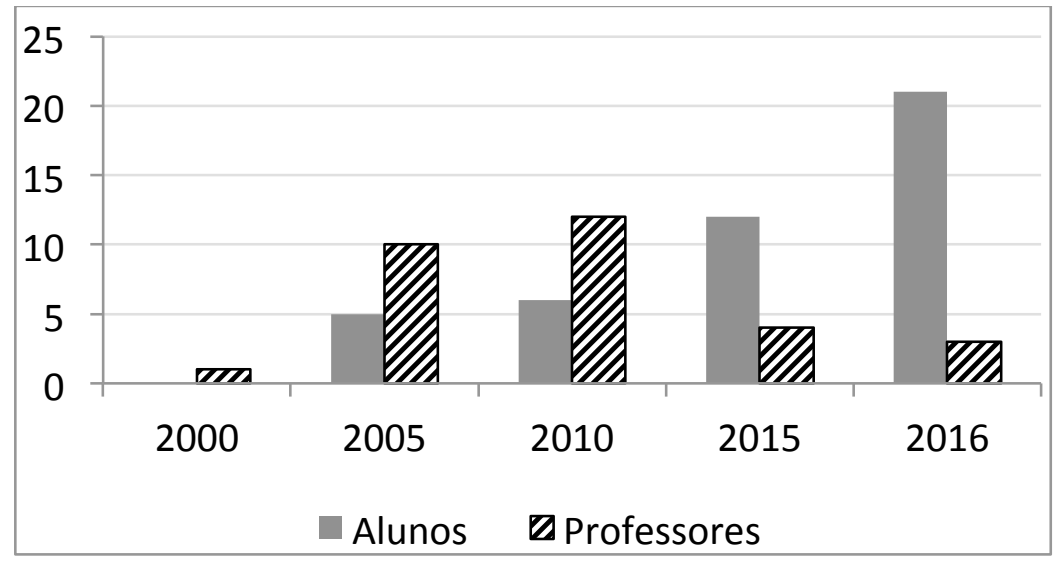

Figura 5: Quantidade de alunos e professores que publicaram periódicos do mestrado em Engenharia de Minas da UFOP (Plataforma Sucupira).

Segundo dados apresentados na Figura 5 as publicações por parte dos professores superam aquelas dos alunos até o ano de 2010. Com o aumento de matriculados, cresceram também as publicações, houve estimulo e incentivo de professores que compreendem a necessidade de formação de novos docentes que ampliaram seus projetos de pesquisas abrindo caminho para mais divulgações.

Desafios futuros do curso incluem aumentar a produção científica dos discentes, acrescentar à matriz curricular disciplinas e ações que desenvolvam a aptidão para a docência, acrescentar ações e disciplinas que contemplem as humanidades, ações de extensão universitária entre outras iniciativas. Tudo isso e muito mais vêm com a objetividade de fortalecer o Plano Nacional de Pós-Graduação (PNPG) que teve sua primeira versão em 1975 e precisa ser colocado em prática.

\subsection{O que diz o Plano Nacional de Pós-Graduação (PNPG)}

Segundo Vogel e Kobaschi (2015) o PNPG 2011-2020 é o sexto plano criado pela CAPES. Os anteriores foram:

I PNPG (1975-1979): que introduziu o planejamento estatal das atividades de Pós Graduação no Brasil, com o objetivo de formar docentes e pesquisadores;

II PNPG (1982-1985): que se preocupou com a institucionalização da avaliação da PósGraduação, processo existente desde 1976, que era, porém, incipiente;

III PNPG (1986-1989): que propôs a subordinação das atividades de Pós-Graduação ao desenvolvimento econômico brasileiro, por meio de sua integração com o Sistema Nacional de Ciência e Tecnologia.

IV PNPG: que não se concretizou como um plano oficial, mas teve suas diretrizes adotadas pela CAPES: ênfase na expansão do sistema, na inserção do Sistema Nacional da PósGraduação e apresentação de propostas de mudanças no processo de avaliação. 
V PNPG (2005-2010): que propôs o aprimoramento do processo de avaliação qualitativa da Pós-Graduação, a preocupação com a solidariedade entre os cursos e seu impacto social, a expansão da cooperação internacional, o combate às assimetrias, a formação de recursos humanos para a inovação tecnológica, a ênfase na formação de docentes para todos os níveis de ensino e a criação de uma nova modalidade de curso: o mestrado profissional.

VI PNPG (2011-2020):tem como objetivo central promover a integração do ensino de pósgraduação com o setor empresarial e a sociedade. Além disso, propõe a organização de uma agenda nacional de pesquisa em torno de temas, a superação das assimetrias e a formação de recursos humanos para empresas e programas nacionais (BRASIL, 2010, p.18). Embora reconheça que a pesquisa é a essência da pós-graduação há, neste plano, forte preocupação com a formação de professores para o ensino médio e básico.

\subsection{Sugestões}

Com o intuito de colocar o PNPG em prática e alavancar a formação de bons professores, sugere-se, ainda com necessárias adequações, as iniciativas:

Criar avaliações realizadas por alunos, semelhante à graduação, gerando ações em acordo com os resultados destas avaliações;

Criar premiações anuais para desempenho de pós-graduandos. Os melhores avaliados teriam apoio financeiro para participação em eventos nacionais da área. Sendo $R \$ 1.500,00$ para eventos nacionais e $\mathrm{R} \$ 3.000,00$ para internacionais relacionados à área da mineração;

Tornar obrigatório o aluno de doutorado lecionar uma disciplina, no mínimo, da área por pelo menos um ano (essa atividade devendo somar 3 créditos no histórico do aluno). Como exemplo, a Pró-Reitoria de Graduação (PROGRAD-UFOP) já permiti o registro de professor colaborador (doutorando) por um ano sem prolabore;

Criar um programa de iniciação científica ou autorizar alunos de pós a orientar nos programa Bic Junior (FAPEMIG) e Bic EM (CNPq) (Lembrando que faltam orientadores para esse programas);

Possibilitar aos doutorandos submeter projetos de extensão e proativa;

Possibilitar a orientação dos alunos de pós-graduação de trabalho final de curso, conferindo-o um até três creditos por orientação;

Buscar interação entre os programas, pois umas das maiores dificuldades é sempre adequar as dissertações e teses as normas ABNT. Deveria ter uma disciplina sobre metodologia. Nem todos alunos de pós fizeram iniciação ou tiveram tal disciplina na graduação.

\section{CONCLUSÕES}

Diretamente relacionado a uma nova era, de mercado de trabalho exigente, que busca a excelência em sua mão-de-obra já dita especializa os cursos de pós-graduação são os principais caminhos para aquele profissional que busca estar preparado para esta realidade. 
Além da formação técnica voltada para atender setores de elevada carência em especialidades, os cursos de Pós-graduação devem formar seres humanos melhores, preocupados com o todo, com a sociedade em que se insere como uma visão superlativa de sustentabilidade.

Os cursos, em especial aqueles que já possuem um embasamento técnico estabelecido, como a referida Pós-graduação em Engenharia Mineral citada neste trabalho, devem voltar seus esforços para a formação de professores, como defende o Plano Nacional de Pós-graduação de 1975 e o atual. Uma carência destes profissionais, em breve, pode se tornar um entrave para o futuro da pós-graduação. Em particular, curso PPGEM-UFOP possui significativo número de bolsistas pesquisadores do CNPq, no entanto nos próximos estarão próximo de suas aposentadorias, pois os professores possuem em média 23 anos de experiência em ensino.

O PPGEM-UFOP atingiu recentemente o objetivo de implantar o doutoramento, mas isso influencia diretamente a produção científica do programa que se deve intensificar como uma maneira de se sustentar. Simultaneamente este avanço abre caminho para uma proximidade maior com o ensino da graduação.

\section{AGRADECIMENTOS}

Agradecemos à Universidade Federal de Ouro Preto, Fundação Gorceix, CAPES, FAPEMIG e ao CNPq.

\section{REFERÊNCIAS}

Plataforma Sucupira.

https://sucupira.capes.gov.br/sucupira/public/informacoes_programa/informacoesPrograma.jsf. Acessado em: 12/05/2016.

CPPGEM-UFOP - Colegiado do Programa de Pós-graduação em Engenharia Mineral da Universidade Federal de Ouro Preto, 2017.

Roque, R. C. M. (1999). Os bandeirantes dos tempos modernos. Dissertação de mestrado, Universidade Federal Fluminense, Rio de Janeiro, Brasil.

Schwartzman, S. (2001). Um espaço para ciência a formação da comunidade científica no Brasil. Ministério da Ciência e Tecnologia.

Vogel, M. J. M, Kobashi, N. Y. (2015). Avaliação da Pós-graduação no Brasil: seus critérios. XVI Encontro Nacional de Pesquisa em Ciência da Informação (XVI ENANCIB).

www.vale.com Acessado em: 29/10/2017. 